\title{
Suppressive Effect of Calcitonin on Intestinal Absorption of Calcium and Phosphorus in Sheep
}

\author{
ToHru MATSUI, Nozomu KURAMITSU, Hideo YANO \\ AND RYOJI KAWASHIMA \\ Faculty of Agriculture, Kyoto University, \\ Kitashirakawa Oiwake-cho, Sakyo-ku, Kyoto-shi, 606
}

\begin{abstract}
This study was carried out to determine the effect of calcitonin on calcium and phosphorus absorption in sheep. Six sheep, which were prepared with a calotid loop and a portal venous catheter, were used. Thyroidectomized sheep were injected intramuscularly with porcine calcitonin at feeding time. Serum calcium concentrations did not change in intact or thyroidectomized sheep after feeding but calcium venoarterial blood differences in thyroidectomized sheep were higher than in intact sheep $8 \mathrm{~h}$ after feeding. Serum calcium concentrations and calcium veno-arterial blood differences were decreased by calcitonin injection into thyroidectomized sheep. Calcitonin injection also reduced the serum phosphorus concentrations. Phosphorus veno-arterial blood differences in thyroidectomized sheep were much less than in intact sheep before feeding although the blood differences were not affected by calcitonin injection in thyroidectomized sheep. The results indicated that calcitonin decreased calcium absorption, which caused a reduction in the serum calcium concentrations, and that calcitonin injection did not affect phosphorus absorption in thyroidectomized sheep.
\end{abstract}

It is well known that calcitonin secreted from the thyroid gland has a hypocalcemic and hypophosphatemic effect in rats (Hirsch et al., 1964) and sheep (Barlet, 1972). The effects are thought to be brought about by the increase in urinary calcium and phosphorus excretion (Barlet, 1972), and the inhibition of bone resorption (Johnston and Deiss, 1966). Though the digestive tract is important for calcium and phosphorus metabolism, the effect of calcitonin on calcium absorption is still controversial and the effect on phosphorus absorption is little known. Krawitt (1967) did not find any significant change in calcium absorption

Received March 17, 1983 after calcitonin injection in rats but Olson et al. (1972) indicated that calcitonin infusion immediately inhibited calcium absorption in rats. However, Swaminathan et al. (1974) suggested that calcitonin indirectly affected calcium absorption because calcium absorption was reduced 2 days after calcitonin infusion in pigs. The experiment was to investigate the effect of calcitonin on calcium and phosphorus absorption in sheep.

\section{Materials and Methods}

Six adult sheep, weighing about $40 \mathrm{~kg}$ were used. Three sheep were thyroidectomized 3 months before the experiment and the others were intact. Every thyroidectomized sheep was injected with $2.5 \mathrm{mg}$ of 
L-thyroxine (Nakarai Chemicals, Ltd., Kyoto) dissolved in corn oil in order to supply thyroid hormone.

Every animal was surgically prepared with a carotid loop and a portal venous catheter. A siliconized catheter was inserted via a branch of the omasum-abomasum vein into the portal vein, then a ligature was tightened around the vessel. The animals were used for the experiment 2 weeks after the surgery. All animals were given a diet containing $0.41 \%$ calcium and $0.40 \%$ phosphorus at a level of $2 \%$ of body weight daily. Water was available at all times. Each thyroidectomized sheep was injected intramuscularly with 1 i.u./kg body weight of porcine calcitonin (Armor Pharmaceutical Company, Ltd., Eastbourne England; 80 i.u./mg protein) dissolved in $16 \%$ gelatin and each intact sheep was injected with the vehicle twice at 5-day intervals at feeding time. On the day before calcitonin administrations, all animals were injected with the vehicle at feeding time.

Blood samples were collected before feeding and $1,2,4$ and $8 \mathrm{~h}$ after feeding on the day of the vehicle injection (TX animals) and the day of calcitonin injection (TX+CT animals) in thyroidectomized sheep. At the same time, blood samples were also obtained from intact sheep.

Hemoglobin content was determined by the method of Drabkin and Austine (1935) to correct water content in blood. Blood serum was separated and stored at $-14^{\circ} \mathrm{C}$ for analysis. Serum calcium concentrations were measured by an atomic absorption spectrophotometry and serum phosphorus concentrations by Gomori's method (1942). Calcium and phosphorus absorption were estimated by a veno-arterial blood difference technique. Statistical differences were evaluated by Student's $t$-test.

\section{Results}

Table 1 shows serum calcium and phosphorus concentrations in arterial blood before feeding. There was no significant difference in serum calcium and phosphorus concentrations between thyroidectomized and intact sheep.

Fig. 1 shows postprandial chages in serum calcium and phosphorus concentrations in arterial blood. Little change was found in serum calcium concentrations in either TX or intact sheep. In TX+CT sheep, serum calcium concentrations began to decrease 1 $\mathrm{h}$ after feeding (calcitonin injection) and
Table 1. Serum Calcium and Phosphorus concentrations before feeding

\begin{tabular}{lccc}
\hline \hline Item & & Intact & Thyroidectomy \\
\hline Calcium & $\mathrm{mg} / 100 \mathrm{ml}$ & $8.14 \pm 0.10$ & $8.44 \pm 0.36$ \\
Phosphorus & $\mathrm{mg} / 100 \mathrm{ml}$ & $2.36 \pm 0.06$ & $2.29 \pm 0.07$ \\
\hline
\end{tabular}

Results are expressed as means \pm SE for 12 samples.

reached $76 \%$ of the initial calcium level 8 $\mathrm{h}$ after feeding.

Through serum phosphorus concentrations significantly $(\mathrm{p}<0.05)$ increased $2 \mathrm{~h}$ after feeding, the concentrations tended to decrease $4 \mathrm{~h}$ after feeding in intact sheep. Serum phosphorus concentrations in TX and TX +CT animals also decreased after feeding. The reduction in serum phosphorus was more remarkable in $\mathrm{TX}+\mathrm{CT}$ animals than in $\mathrm{TX}$ and intact ones.

Fig. 2 shows differences in veno-arterial blood calcium and phosphorus. A significant $(p<0.05)$ increase in calcium absorption was found in TX animals $8 \mathrm{~h}$ after feeding and calcium absorption was decreased significantly $(\mathrm{p}<0.01)$ in $\mathrm{TX}+\mathrm{CT}$ animals.

Differences in veno-arterial blood phosphorus were less in TX and TX $+\mathrm{CT}$ animals than in intact ones before feeding. The differences in phosphorus absorption between thyroidectomized and intact sheep became smaller after feeding whether the thyroidectomized sheep were injected with calcitonin or not.

\section{Discussion}

It was observed in the following experiment that serum calcitonin concentrations, which were measured by a radioimmunoassay using porcine calcitonin, were increased by an intravenous calcuim load in intact sheep but were undetectable $(<1 \mathrm{ng} / \mathrm{ml})$ before and after the calcium load in thyroidectomized sheep (T. Matsui, unpublished data). Therefore the calcitonin secretion might be negligible in thyroidectomized sheep. 


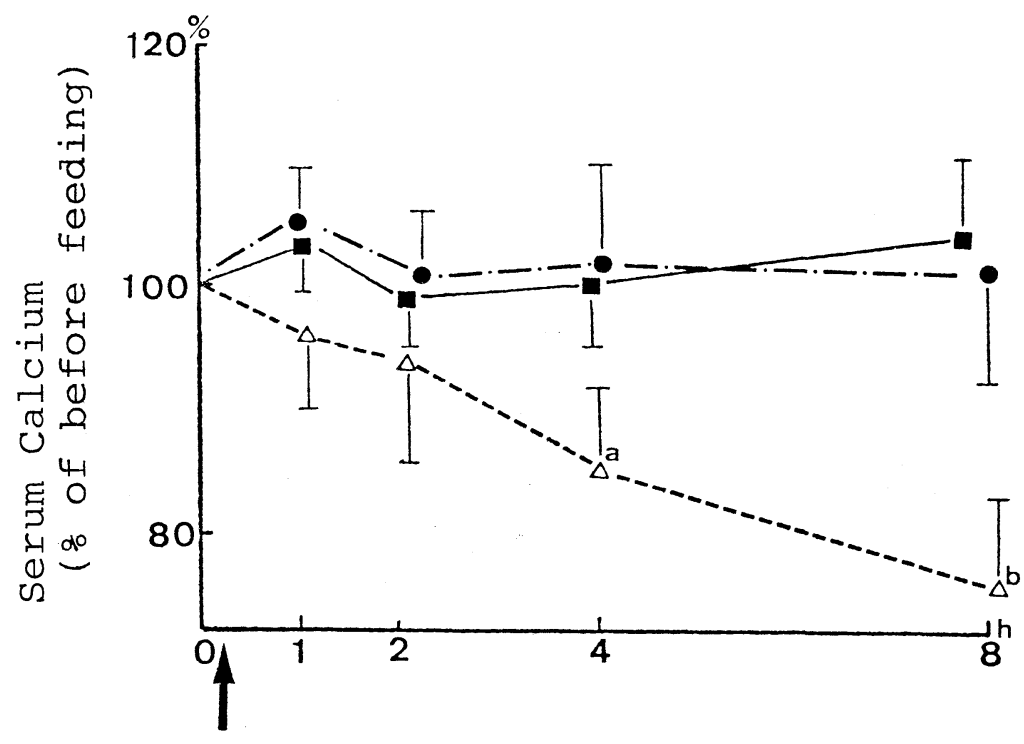

Fig. 1. Changes in serum calcium and phosphorus concentrations in arterial blood after feeding in intact ( $\square$ ), TX (O) and $\mathrm{TX}+\mathrm{CT}(\triangle)$ sheep. TX animals are thyroidectomized sheep and TX $+\mathrm{CT}$ animals are thyroidectomized sheep injected with porcine calcitonin. TX and intact sheep were injected with the vehicle. Values are epressed as \% of before feeding. Vertical bars represent mean $\pm \mathrm{SE}$ for 12 samples in intact sheep and for 6 samples in TX and TX + CT sheep. Significant differences from intact sheep were evaluated by Student's $t$-test at $\mathrm{p}<0.05$ (a) or $\mathrm{p}<0.01$ (b). Arrows indicate feeding and calcitonin or vehicle injection.

The results showing that serum calcium concentrations were decreased by calcitonin administration were in agreement with the observation by Barlet (1972). It is interesting that there was little difference in serum calcium concentrations between TX and intact sheep though calcium absorption in TX sheep was greater than in intact sheep. It was indicated that urinary calcium excretion was increased by thyroidectomy in rats
(Talmage and Grubb, 1977) and sheep (Matsui et al., 1983). The increase in urinary calcium excretion may counteract tne increase in serum calcium concentrations after feeding in TX sheep.

The data showing that calcium absorption in TX animals was greater than in intact ones $8 \mathrm{~h}$ after feeding and calcium absorption was immediately decreased by calcitonin injection showed that calcitonin directly 

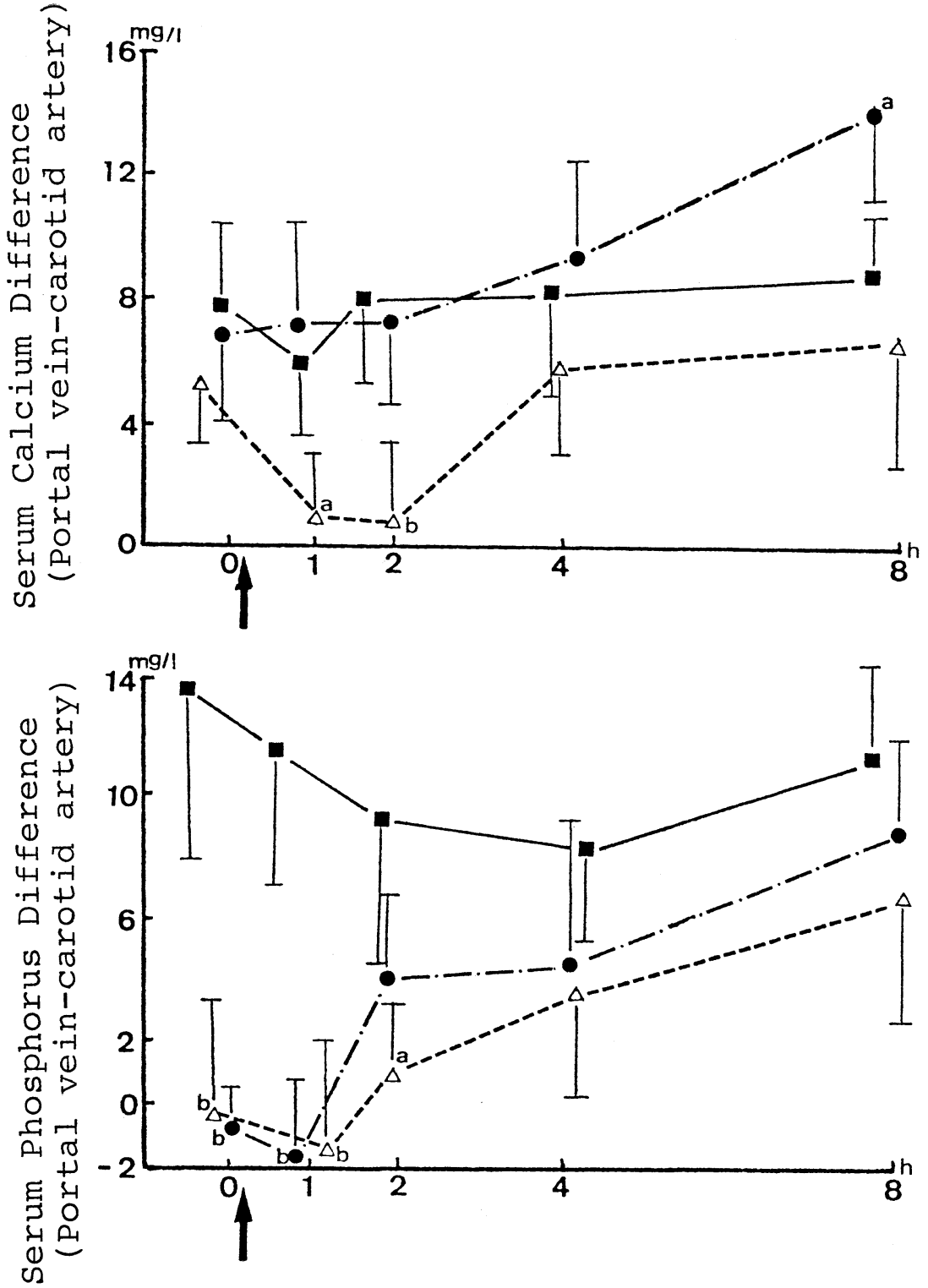

Fig. 2. Portal venous-carotid arterial blood differences in calcium and phosphorus in intact $(\square)$, TX $(O)$ and TX $+\mathrm{CT}(\triangle)$ sheep. TX animals are thyroidectomized sheep and TX+CT animals are thyroidectomized sheep injected with porcine calcitonin. TX and intact animals were injected with the vehicle. Values are corrected by ratios of portal venous-carotid arterial blood hemoglobin content. Vertical bars represent mean $\pm \mathrm{SE}$ for 12 samples in intact and for 6 samples in $\mathrm{TX}$ and $\mathrm{TX}+\mathrm{CT}$ sheep. Significant differences from values for intact sheep were evaluated by Student's $t$ test at $\mathrm{p}<0.05$ (a) or $\mathrm{p}<0.01$ (b). Arrows indicate feeding and calcitonin or vehicle injection. 
influenced calcium absorption in sheep. Olson et al. (1972) also found acute reduction of calcium absorption by calcitonin infusion using an in-vitro rat intestinal perfusion technique. However, Swaminathan et al. using a Thiry-Vella loop technique in swine (1974) reported that calcitonin had no direct effect on calcium absorption because calcium absorption began to be reduced 2 days after calcitonin infusion. The difference between these results may be due to the technique used in estimating calcium absorption. In the Thiry-Vella loop technique, calcium absorption was measured by the disappearance of calcium from the intestinal lumen. On the other hand, calcium absorption was estimated by the outflow of calcium to the portal vein using the veno-arterio blood difference method and the in-vitro intestinal perfusion technique. It is suggested that calcitonin may directly inhibit the influx of calcium from the intestinal mucosa to the blood of the portal vein over a short term and then indirectly reduce the efflux of calcium from the intestinal lumen over a long term.

Yamaguchi (1979) demonstrated the increase in calcium excretion via bile when calcitonin was injected into rats. It is possible that calcium absorption from the gut was depressed but calcium concentrations in the intestinal lumen were increased by the calcitonin injection in sheep.

Serum phosphorus concentrations were temporarily elevated $2 \mathrm{~h}$ after feeding in intact sheep but the elevation of serum phosphorus was not found in TX and TX+ CT sheep. The increase in serum phosphorus levels might be caused by phosphorus absorption from the gut because phosphorus absorption was much greater in intact sheep than in TX and TX+CT ones. Calcitonin injection lowered serum phosphorus concentrations. A hypophosphatemic effect of calcitonin was reported in sheep (Barlet, 1972).

The finding that phosphorus absorption was not different between $\mathrm{TX}$ and $\mathrm{TX}+\mathrm{CT}$ animals indicated that calcitonin did not seriously affect phosphorus absorption in sheep. However, phosphorus absorption in thyroidectomized sheep was much less than in intact ones before feeding. The reason why there was so little phosphorus absorption before feeding and that it tended to increase after feeding in thyroidectomized sheep was not clear. However it is well established that salivary phosphorus plays an important role in phosphorus metabolism of ruminants (Kay, 1966). It is possible that the capacity for phosphorus absorption was not different between thyroidectomized and intact sheep though the amount of phosphorus inflow via saliva was less in thyroidectomized sheep than in intact ones.

\section{Acknowledgement}

This work was supported in part by the Nisshin Seifun Foundation.

\section{References}

Barlet, J. P. (1972). Effect of porcine, salmon and human calcitonin on urinary excretion of some electrolytes in sheep. $J$. Endocrinol. 55, 153-161.

Drabkin, D. L. and J. H. Austin (1935). A technique for the analysis of undiluted blood and concentrated hemoglobin solution. J. Biol. Chem. 112, 105-115.

Gomori, G. (1942). A modification of the colorimetric phosphorus determination for use with the photoelectric colorimeter. J. Lab. Clin. Med. 27, 955-960.

Hirsch, P. F. and P. L. Munson (1964). Thyrocalcitonin; hypocalcemic hypophosphatemic principal of the thyroid gland. Science 146, 412-413.

Johnston, C. C. Jr. and W.P. Deiss, Jr. (1966). An inhibitory effect of thyrocalcitonin on calcium release in vivo and bone metabolism in vitro. Endocrinology. 78, 1139-1143.

Kay, R. N. B. (1966). The influence of salvia on digestion in ruminants. World Review of Nutrition and Dietetics. 6, 292-325.

Krawitt, E. L. (1967). Effect of thyrocalcitonin on duodenal calcium transport. 125, 1084-1086.

Matsui, T., H. Yano and R. Kawashima (1983). 
Effect of thyroidectomy and thyroparathyroidectomy on serum calcium, phosphorus and hydroxyproline concentration in sheep. Jap. J. Zootech. Sci. 54, 232-238.

Olson, E. B., Jr., H. F. Deluca and J. T. Potts, Jr. (1972). Calcitonin inhibition of vitamin D induced intestinal calcium absorption. Endocrinology 90, 151-157.

Swaminathan, R., J. Ker and A. D. Care (1974).
Calcitonin and intestinal calcium absorption. $J$. Endocrinol. 61, 83-94.

Talmage, R. V. and S. T. Grubb (1977). The influence of endogenous calcitonin on daily urinary calcium excretion. Endocrinology 101, 1351-1357.

Yamaguchi, M. (1979). Effects of calcitonin and other hormones on bile calcium excretion in thyroparathyroidectomized rats. Endocrinol. Japon. 26, 111-115. 